Originally published as:

Pilger, D., Hauser, A., Kuecherer, C., Mugenyi, K., Kabasinguzi, R., Somogyi, S., Harms, G., Kunz, A.

Minor drug-resistant HIV type-1 variants in breast milk and plasma of HIV type-1-infected Ugandan women after nevirapine single-dose prophylaxis

(2011) Antiviral Therapy, 16 (1), pp. 109-113.

DOI: 10.3851/IMP1698

This is the author's version of a work accepted for publication by International Medical Press. A definitive version was published in Antiviral Therapy, (16, 1), 29 November 2010, ( 2010 International Medical Press." 


\title{
Minor drug-resistant HIV type-1 variants in breast milk and plasma of HIV type-1-infected Ugandan women after nevirapine single-dose prophylaxis
}

\author{
Daniel Pilger ${ }^{1}$, Andrea Hauser ${ }^{1,2}$, Claudia Kuecherer ${ }^{2}$, Kizito Mugenyi ${ }^{3}$, Rose Kabasinguzi ${ }^{3}$, Sybille \\ Somogyi ${ }^{2}$, Gundel Harms ${ }^{1, *}$, Andrea Kunz $^{1}$ \\ ${ }^{1}$ Institute of Tropical Medicine and International Health, Charité - Universitâtsmedizin Berlin, Berlin, \\ Germany \\ ${ }^{2}$ Project HIV Variability and Molecular Epidemiology, Robert Koch Institute, Berlin, Germany \\ ${ }^{3} \mathrm{MoH} / \mathrm{GTZ}$ PMTCT Project western Uganda, Fort Portal, Uganda
}

*Corresponding author e-mail: gundel.harms@charite.de

\begin{abstract}
Background: Nevirapine single-dose (NVP-SD) reduces mother-to-child transmission of HIV type-1 (HIV-1), but frequently induces resistance mutations in the HIV-1 genome. Little is known about drugresistant HIV-1 variants in the breast milk of women who have taken NVP-SD.

Methods: Blood and breast milk samples of 39 HIV-1-infected Ugandan women were taken 6-12 weeks after NVP-SD intake. Samples were analysed by population sequencing and allele-specific real-time PCR (AS-PCR) with detection limits for NVP-resistant HIV-1 variants (K103N and Y181C) of $<1 \%$ of the total viral population.

Results: AS-PCR results for both plasma and breast milk were obtained for 19 women who constituted the final study group (HIV-1 subtype frequencies were $A 1 n=11, D n=5, G n=2$ and $C n=1$ ). A total of 7 (37\%) and 10 (53\%) women carried NVP-resistant virus in breast milk and plasma, respectively. Overall, 71\% (5/7) women with NVP-resistant HIV-1 in breast milk displayed $>1$ drug-resistant variant. Resistance in breast milk was higher at week 6 (6/13 samples [46\%]) compared with week 12 (1/6 samples [17\%]). In total, 10 drug-resistant populations harbouring the K103N and/or Y181C mutation were detected in the 19 breast milk samples; 7 (70\%) were caused by resistant minorities ( $<5 \%$ of the total HIV-1 population). In the four women with drug-resistant virus in both plasma and breast milk, the mutation patterns differed between the two compartments.
\end{abstract}

Conclusions: Minor populations of drug-resistant HIV-1 were frequently found in breast milk of Ugandan women after exposure to NVP-SD. Further studies need to explore the role of minor drugresistant variants in the postnatal transmission of (resistant) HIV-1.

\section{Introduction}

Mother-to-child transmission of HIV is a major concern in sub-Saharan Africa, and postnatal HIV infection through breastfeeding is estimated to account for almost one-third to one-half of all HIV infections in infants and young children [1]. Nevirapine single-dose (NVP-SD) is still widely used to reduce the transmission of HIV type-1 (HIV-1) from the mother to the child in resource-limited settings, although it is associated with a high incidence of NVP-resistant HIV-1 [2-11]. To date, most studies have focused on the development of NVP-resistant virus in plasma, and it has been shown that 1975\% of women exposed to NVP-SD harbour NVP-resistant HIV-1 variants in their blood [3-11]. Although the proportion of resistant virus in plasma fades over time, minor drug-resistant populations can persist for long periods in this compartment $[5,8,9]$. However, little is known about the emergence and persistence of NVP-resistant HIV-1 variants in breast milk after NVP-SD and to what extent the mutation patterns differ from plasma. This is of great importance as breastfeeding is common in many developing countries where NVP-SD is used and the presence of resistant virus in breast milk might put children at risk of acquiring drug-resistant HIV-1 through breast milk [12]. 
So far, only three studies have determined the emergence of NVP-resistant virus in breast milk following intake of NVP-SD. Two studies analysed breast milk of women infected with HIV-1 subtype C and showed that HIV-1 variants in breast milk emerged frequently and often differed in sequences from resistant HIV-1 variants in plasma [13,14]. Recently, a third study analysed breast milk samples of women mainly infected with HIV-1 subtypes A and D using a population-based approach with a detection limit for drug-resistant HIV-1 variants of approximately $20 \%$ [15].

Here, we describe the emergence of NVP-resistant HIV-1 variants in breast milk and plasma of Ugandan women predominantly infected with HIV-1 subtypes A and D using highly sensitive allelespecific real-time PCR (AS-PCR), capable of detecting minor drug-resistant variants representing $<1 \%$ of the total viral population.

\section{Methods}

We conducted an observational cohort study that was approved by the national ethical committee of Uganda (National Council of Science and Technology). HIV-1-positive pregnant women enrolled in the national prevention of mother-to-child transmission programme were recruited at Fort Portal Hospital (Fort Portal, western Uganda) after they had given informed written consent [16]. The women had taken NVP-SD $(200 \mathrm{mg})$ at the onset of labour following the HIVNET 012 protocol [2]. None of the participating women received any other antiretroviral drugs aside from NVP-SD before or during the study period. Plasma and breast milk samples were collected at delivery (baseline) and at 1, 2, 6 and 12 weeks postpartum. Participants were included in the final analysis if plasma and breast milk samples from delivery were available and follow-up samples at week 6 or 12 were amplifiable. Whenever the baseline sample was amplifiable it was used as individual threshold for the detection of NVP-resistant variants as described by Hauser et al. [17]; otherwise a cutoff derived from plasmid mixtures was applied. HIV-1 variants with the K103N and/or Y181C mutation in the pol gene were detected and quantified by two-step AS-PCR assays, as previously described [17]; the detection limits for the three mutations as estimated from plasmid controls were $0.019 \%$ (K103N:AAC), $0.013 \%$ (K103N:AAT) and 0.29\% (Y181C:TGT). To compensate for the expected lower viral loads in breast milk, the sample volume for RNA extraction was increased sixfold $(3 \mathrm{ml})$ as compared with plasma $(0.5 \mathrm{ml})$. Viral RNA was extracted (QIAamp Viral RNA Mini Kit; Qiagen GmbH, Hilden, Germany) and reverse transcribed (SuperScript II RT; Invitrogen, Karlsruhe, Germany) according to the manufacturer's recommendations. Viral loads were determined in the outer quantitative PCR (644 base pair amplimer with coordinates 2613-3256 of HXB2; GenBank accession number K03455) using a quantified virus stock (NL4.3) as standard. The lower limit of detection for viral load was 650 copies $/ \mathrm{ml}$; that is, the input of $3 \mathrm{ml}$ breast milk allowed the quantification of viral loads down to 220 copies $/ \mathrm{ml}$. For population-based sequencing of the 644 base pair product (comprising the codons 21236 of the reverse transcriptase), the automated sequencer 3130xl Genetic Analyzer (Applied Biosystems, Darmstadt, Germany) and the B-, D- and G-sequencing primers of the ViroSeq HIV-1 Genotyping System version 2.0 (Abbott GmbH \& Co. KG, Wiesbaden, Germany) were used [17]. The sequences were subtyped with the REGA HIV-1 subtyping tool [18] or by the neighbour-joining method (PHYLIP package [19]). A neighbour-joining tree was constructed by aligning the pol sequences with 131 subtype reference sequences from the pol subtype reference alignment 2008 [20] using the CLUSTALW programme implemented in BioEdit version 7.0.5 [21]. All samples were measured at least in duplicates together with no template- and HIV-1-negative controls. A neighbourjoining phylogenetic tree was calculated to control for potential sample mix-up. Sequences derived from plasma and breast milk from one patient should colocalize in clades, whereas independent HIV-1 strains should not cluster together.

\section{Results}

Blood samples and breast milk samples of 39 women were available. For 20 (51\%) women, the analyses of breast milk samples were PCR-negative because viral loads were below the detection level of the assays. Samples from these women were omitted from the final analysis, and the remaining 19 women constituted the final study group. The median age was 25 years (interquartile range [IQR] 19-29) and the median parity was 2 (IQR 1-5). A total of 11 (58\%) women were infected with subtype A1, 5 (26\%) with subtype D, $2(11 \%)$ with subtype $G$ and $1(5 \%)$ with subtype C. The median viral load in breast milk was 1,100 copies/ml (IQR 500-2,800 copies/ml; Table 1). 
Overall, 7 (37\%) women harboured drug-resistant HIV-1 in breast milk and 10 (53\%) women in plasma (Table 1). NVP-resistant virus in breast milk was detected in $5 / 7$ subtype $A 1,0 / 5$ subtype $D, 1 / 2$ subtype $G$ and $1 / 1$ subtype $C$ samples, respectively. A total of 13 breast milk samples were collected 6 weeks and 6 were collected 12 weeks after NVP-SD intake. At week 6, drug-resistant virus was detected in 6/13 (46\%) breast milk samples, whereas at week 12, 1/6 (17\%) breast milk samples exhibited NVP-resistant HIV-1. In total, 10 and 13 drug-resistant populations carrying the K103N or Y181C mutation were detected in breast milk and plasma samples, respectively. Among these, 7 $(70 \%)$ in the breast milk and $5(38 \%)$ in the plasma were present as resistant minor variants defined as $<5 \%$ of the total viral population.

In $4(21 \%)$ women, drug-resistant virus was identified in both breast milk and plasma. In these women (patient numbers 2, 4, 6 and 12; Table 1), the mutation patterns differed between the two compartments. In 5/7 (71\%) women (patient numbers 2, 4, 6, 12 and 15; Table 1) with drug-resistant virus in breast milk, $>1$ NVP-resistant HIV-1 variants were found in the same breast milk sample.

All samples identified as wild-type HIV-1 (K103 and Y181) by AS-PCR yielded wild-type results by population-based sequencing. Whenever population-based sequencing detected HIV-1 variants carrying the $\mathrm{K} 103 \mathrm{~N}$ and/or Y181C mutation, the AS-PCR assays confirmed the presence of mutations. Population-based sequencing with its much lower sensitivity failed to detect 15 drug-resistant variants identified by the AS-PCR assays (Table 1 ). By contrast, population-based sequencing detected other NVP-associated resistance mutations, such as V106A and G190A in breast milk; however, it did no identify additional women with drug-resistant HIV-1 in breast milk, as all of these women carried the $\mathrm{K} 103 \mathrm{~N}$ and/or Y181C mutation, which had already been detected by the AS-PCR assays. The neighbour-joining tree topology did not reveal any indication of sample mix-up. Only HIV-1 sequences derived from plasma and breast milk from the same individual were closely related (supported by high bootstrap values) in contrast to sequences derived from independent infections (CK et al., data not shown).

\section{Discussion}

Our findings confirm previous studies reporting high frequencies of drug-resistant virus in breast milk of NVP-SD-exposed pregnant women infected with HIV-1 [13-15]. Additionally, we found that most of the detected resistance mutations were present as minor drug-resistant variants accounting for $<5 \%$ of the total viral population. The effect of such minor variants on the transmission of drug-resistant HIV-1 to the infant through breastfeeding is so far unknown, and needs further exploration.

Although it has been shown that minor drug-resistant variants can persist in plasma for prolonged periods $[5,8,9]$, their persistence in breast milk is not well characterized. Knowledge of the persistence of drug-resistant variants is important to better estimate the risk of transmission of drug-resistant HIV-1 through breastfeeding. In this study, using highly-sensitive AS-PCR assays, we found that $46 \%$ and $17 \%$ of all women harboured drug-resistant HIV-1 variants in their breast milk at 6 and 12 weeks after NVP-SD intake, respectively. These findings suggest a fading of NVP-resistant virus in breast milk over time as it was shown for plasma [5,8,9]. Recently in Uganda, Hudelson et al. [15] detected NVPresistant virus in samples of $40 \%$ of women 4 weeks after NVP-SD intake using population-based sequencing. It can be assumed that the frequency of drug-resistant variants would have been even higher if they had applied a more sensitive method, such as AS-PCR assays.

The percentage of women harbouring drug-resistant variants in our study was lower compared with studies analysing HIV-1 subtype-C-infected women [13,14]. Lee et al. [14] found drug-resistant virus in $65 \%$ of breast milk samples of women infected with HIV-1 subtype C 8 weeks after NVP-SD exposure using a population-based approach. Similarly, drug-resistant viruses in plasma have been shown to emerge at higher frequencies in subtype $C$ as compared with subtype $A$ and $D[4,11]$, which also appears to be true for breast milk.

The persistence of drug-resistant variants in breast milk over months could have an effect on the transmission of drug-resistant HIV-1. As the risk of infection increases with the duration of breastfeeding [22], it is conceivable that prolonged breastfeeding increases the risk of acquiring drugresistant HIV-1. 
To estimate the risk of acquiring drug-resistant virus through breastfeeding, the emergence of drugresistant virus in the breast milk compartment has to be understood. Our neighbour-joining analysis showed a close relationship between virus populations found in plasma and in breast milk of the same individual; however, viral strains differed with respect to resistance mutations in the two compartments. Different mutation patterns in breast milk and plasma is a consistent finding $[13,14,23]$. Furthermore, HIV-1 mutation patterns can also differ between samples from the right and left breast of the same women [14]. While it has been shown that a short-course of combivir (zidovudine and lamivudine) over 1 week can prevent the formation of NVP resistances in plasma after NVP-SD intake [24], it is not known whether this holds true for other compartments, that is, for breast milk.

Caution has to be taken when generalizing the results of this study. One-half of the initially enrolled women could not be included into the final resistance analysis because we did not obtain PCR amplificates. This constitutes a common problem in the analysis of breast milk because the viral load in breast milk is considerably lower than in plasma [25]. The real lower limits of detection for the K103N and Y181C variants using AS-PCR assays depend on the respective viral load. Therefore the sensitivity of the assays for breast milk samples with low viral load is reduced compared with the theoretical sensitivity as determined by plasmid controls. Thus, the presence of minor variants in breast milk is prone to be underestimated.

In conclusion, we have shown that minor drug-resistant variants frequently emerge in breast milk of Ugandan women infected with HIV-1 who took NVP-SD. Considering that breastfeeding is still the only option for many women in areas with a high HIV-1 prevalence, the exact role of minor drug-resistant variants in the postnatal transmission of HIV-1 should be subject to further investigations.

\section{Acknowledgements}

We are grateful to the women who have participated in this study. The laboratory support of Silver Mashate is highly acknowledged.

This study was supported by the German Ministry for Economic Cooperation and Development through the project PN 01.2029.5 (Prevention of Mother-to-Child Transmission of HIV) and by a grant of the HW \& J Hector Stiftung, Germany.

\section{Disclosure statement}

The authors declare no competing interests.

\section{References}

1. World Health Organization. HIV transmission though breastfeeding: a review of available evidence. Geneva: World Health Organization 2004.

2. Guay LA, Musoke P, Fleming T, et al. Intrapartum and neonatal single-dose nevirapine compared with zidovudine for prevention of mother-to-child transmission of HIV-1 in Kampala, Uganda: HIVNET 012 randomised trial. Lancet 1999; 354: 795-802.

3. Eshleman SH, Guay LA, Mwatha A, et al. Comparison of nevirapine (NVP) resistance in Ugandan women 7 days vs. 6-8 weeks after single-dose NVP prophylaxis: HIVNET 012. AIDS Res Hum Retroviruses 2004; 20: 595-599.

4. Eshleman SH, Hoover DR, Chen S, et al. Nevirapine (NVP) resistance in women with HIV-1 subtype C, compared with subtypes A and D, after the administration of single-dose NVP. J Infect Dis 2005; 192: 30-36.

5. Flys TS, Donnell D, Mwatha A, et al. Persistence of K103N-containing HIV-1 variants after singledose nevirapine for prevention of HIV-1 mother-to-child transmission. $J$ Infect Dis 2007; 195: 711-715. 
6. Flys TS, McConnell MS, Matovu F, et al. Nevirapine resistance in women and infants after first versus repeated use of single-dose nevirapine for prevention of HIV-1 vertical transmission. $\mathrm{J}$ Infect Dis 2008; 198: 465-469.

7. Johnson JA, Li JF, Morris L, et al. Emergence of drug-resistant HIV-1 after intrapartum administration of single-dose nevirapine is substantially underestimated. J Infect Dis 2005; 192: 16-23. 8. Loubser S, Balfe P, Sherman G, Hammer S, Kuhn L, Morris L. Decay of K103N mutants in cellular DNA and plasma RNA after single-dose nevirapine to reduce mother-to-child HIV transmission. AIDS 2006; 20: 995-1002.

9. Palmer S, Boltz V, Martinson N, et al. Persistence of nevirapine-resistant HIV-1 in women after single-dose nevirapine therapy for prevention of maternal-to-fetal HIV-1 transmission. Proc Natl Acad Sci U S A 2006; 103: 7094-7099.

10. Lockman S, McIntyre JA. Reduction of HIV-1 drug resistance after intrapartum single-dose nevirapine. Lancet 2007; 370: 1668-1670.

11. Flys TS, Chen S, Jones DC, et al. Quantitative analysis of HIV-1 variants with the K103N resistance mutation after single-dose nevirapine in women with HIV-1 subtypes A, C, and D. J Acquir Immune Defic Syndr 2006; 42: 610-613.

12. Moorthy A, Gupta A, Bhosale R, et al. Nevirapine resistance and breast milk HIV transmission: effects of single and extended-dose nevirapine prophylaxis in subtype C HIV-infected infants. PLoS ONE 2009; 4: e4096

13. Kassaye $\mathrm{S}$, Lee $\mathrm{E}$, Kantor $\mathrm{R}$, et al. Drug resistance in plasma and breast milk after single-dose nevirapine in subtype C HIV type 1: population and clonal sequence analysis. AIDS Res Hum Retroviruses 2007; 23: 1055-1061.

14. Lee EJ, Kantor R, Zijenah L, et al. Breast-milk shedding of drug-resistant HIV-1 subtype C in women exposed to single-dose nevirapine. J Infect Dis 2005; 192: 1260-1264.

15. Hudelson SE, McConnell MS, Bagenda D, et al. Emergence and persistence of nevirapine resistance in breast milk after single dose nevirapine administration. AIDS 2010; 24: 557-561.

16. Kunz A, Frank M, Mugenyi K, et al. Persistence of nevirapine in breast milk and plasma of mothers and their children after single-dose administration. J Antimicrob Chemother 2009; 63: 170-177. 17. Hauser A, Mugenyi K, Rose K, et al. Detection and quantification of minor HIV-1 variants harbouring $\mathrm{K} 103 \mathrm{~N}$ and $\mathrm{Y} 181 \mathrm{C}$ resistance mutations in subtype $A$ and $D$ isolates by allele-specific realtime PCR. Antimicrob Agents Chemother 2009; 53: 2965-2973.

18. de Oliveira T, Deforche K, Cassol S, et al. REGA HIV-1 automated subtyping tool. (Accessed 15 November 2010.) Available from http://jose.med.kuleuven.be/genotypetool/html/subtypinghiv.html 19. PHYLIP. (Accessed 15 November 2010.) Available from

http://evolution.gs.washington.edu/phylip.html

20. Los Alamos H. IV Database. (Accessed 15 November 2010.) Available from

http://www.hiv.lanl.gov/content/sequence/NEWALIGN/align.html

21. BioEdit. Version 7.0.5. (Accessed 15 November 2010.) Available from

http://www.mbio.ncsu.edu/BioEdit/bioedit.html

22. John-Stewart G, Mbori-Ngacha D, Ekpini R, et al. Breast-feeding and transmission of HIV-1. J

Acquir Immune Defic Syndr 2004; 35: 196-202.

23. Andreotti M, Guidotti G, Galluzzo CM, et al. Resistance mutation patterns in plasma and breast milk of HIV-infected women receiving highly-active antiretroviral therapy for mother-to-child transmission prevention. AIDS 2007; 21: 2360-2362.

24. McIntyre JA, Hopley M, Moodley D, et al. Efficacy of short-course AZT plus 3TC to reduce nevirapine resistance in the prevention of mother-to-child HIV transmission: a randomized clinical trial. PLoS Med 2009; 6: e1000172

25. Rousseau CM, Nduati RW, Richardson BA, et al. Longitudinal analysis of human immunodeficiency virus type 1 RNA in breast milk and of its relationship to infant infection and maternal disease. J Infect Dis 2003; 187: 741-747. Medline doi:10.1086/374273 
Table 1. Drug-resistant HIV-1 variants in plasma and breast milk detected by population-based sequencing and AS-PCR 6-12 weeks after NVP-SD intake

\begin{tabular}{|c|c|c|c|c|c|c|c|c|c|c|c|c|c|c|c|}
\hline \multirow[b]{2}{*}{$\begin{array}{l}\text { Patient } \\
\text { number }\end{array}$} & \multirow[b]{2}{*}{ HIV-1subtype } & \multicolumn{2}{|c|}{$\begin{array}{l}\text { Viral load, } \\
\text { copies } / \mathrm{ml}\end{array}$} & \multicolumn{2}{|c|}{$\begin{array}{l}\text { Sample collection, } \\
\text { weeks after } \\
\text { IVPP-SD }\end{array}$} & \multicolumn{2}{|c|}{$\begin{array}{l}\text { IIVP-associated } \\
\text { resistant mutations } \\
\text { by population } \\
\text { sequencing }\end{array}$} & \multicolumn{2}{|c|}{$\begin{array}{l}\text { K1031I (AAC) by } \\
\text { AS-PCR, \% }\end{array}$} & \multicolumn{2}{|c|}{$\begin{array}{l}\text { K10311 (AAT) by } \\
\text { AS-PCR, \% }\end{array}$} & \multicolumn{2}{|c|}{$\begin{array}{l}\text { Y181C (TGT) by } \\
\text { AS-PCR, \% }\end{array}$} & \multicolumn{2}{|c|}{ HVP resistance } \\
\hline & & Plasma & $\begin{array}{l}\text { Breast } \\
\text { milk }\end{array}$ & Plasma & $\begin{array}{l}\text { Breast } \\
\text { milk }\end{array}$ & Plasma & $\begin{array}{l}\text { Breast } \\
\text { milk }\end{array}$ & Plasma & $\begin{array}{l}\text { Breast } \\
\text { milk }\end{array}$ & Plasma & $\begin{array}{l}\text { Breast } \\
\text { milk }\end{array}$ & Plasma & $\begin{array}{l}\text { Breast } \\
\text { milk }\end{array}$ & Plasma & $\begin{array}{l}\text { Breast } \\
\text { milk }\end{array}$ \\
\hline 1 & A1 & 96,000 & 420 & 6 & 6 & wt & wt & wt & wt & wt & wt & wht & wt & No & No \\
\hline 2 & A1 & 120,000 & 7,400 & 6 & 6 & $\mathrm{~K} 103 \mathrm{~N}$ & $\mathrm{~V} 106 \mathrm{~A}$ & 15.6 & 9.7 & 0.34 & wt & wt & wt & Yes & Yes \\
\hline 3 & D & 91,000 & 3,400 & 6 & 6 & wt & ND & 6.8 & wt & 0.22 & wht & 0.62 & wt & Yes & No \\
\hline 4 & G & 9,000 & 2,600 & 12 & 12 & $\mathrm{~K} 103 \mathrm{~N}$ & $\begin{array}{l}\text { K103N, } \\
\text { G190A }\end{array}$ & 54.8 & 26.9 & $w t$ & 0.03 & $w t$ & wt & Yes & Yes \\
\hline 5 & D & 95,000 & 14,000 & 6 & 6 & wt & wt & wt & wt & wt & wt & wt & wt & No & No \\
\hline 6 & c & 21,000 & 2,800 & 12 & 6 & $\mathrm{Y} 181 \mathrm{C}$ & $\mathrm{V} 106 \mathrm{~A}$ & wt & 0.67 & wt & 0.04 & 9.8 & wt & Yes & Yes \\
\hline 7 & G & 22,000 & 360 & 12 & 12 & wt & ND & wt & wt & wt & wt & 0.5 & wt & Yes & No \\
\hline 8 & A1 & 19,000 & 1,160 & 6 & 12 & wt & wt & wt & wt & wt & wh & wt & wt & No & No \\
\hline 9 & A1 & 11,000 & 5,000 & 6 & 6 & ND & wt & wt & wt & wt & wt & wt & 2.6 & No & Yes \\
\hline 10 & A1 & 3,500 & 1,100 & 12 & 12 & $\mathrm{~V} 106 \mathrm{~A}$ & ND & wh & wt & wt & wh & wt & wt & Yes & No \\
\hline 11 & D & 2,000 & 500 & 6 & 6 & wt & ND & $w t$ & wt & wt & wt & wt & wt & No & No \\
\hline 12 & A1 1 & 200,000 & 1,340 & 6 & 6 & $\begin{array}{l}\text { K103Na, } \\
\text { Y181C }\end{array}$ & $\begin{array}{l}\mathrm{K} 101 \mathrm{E}, \\
\mathrm{K} 103 \mathrm{~N}\end{array}$ & 26.1 & 100 & 35 & 0.04 & 100 & $w t$ & Yes & Yes \\
\hline 13 & A1 & 4,700 & 340 & 12 & 12 & wh & wt & wt & wht & wt & wht & wt & wt & No & No \\
\hline 14 & D & 4,600 & 660 & 6 & 6 & $\mathrm{Y} 188 \mathrm{C}$ & wt & 19.2 & wt & wt & wt & wt & wt & Yes & No \\
\hline 15 & A1 & 12,000 & 1,000 & 12 & 6 & ND & K101E & wt & wt & wt & 0.02 & wt & wt & No & Yes \\
\hline 16 & A1 & 5,000 & 340 & 6 & 6 & wt & $w t$ & wt & wt & wt & wt & wt & wt & No & No \\
\hline 17 & A1 & 5,400 & 1,400 & 12 & 12 & wt & wt & 1.2 & wt & wt & wt & wt & wt & Yes & No \\
\hline 18 & A1 & 8,100 & 600 & 6 & 6 & wt & wt & wt & wt & wt & wt & wt & 1.6 & No & Yes \\
\hline 19 & D & 3,600 & 540 & 6 & 6 & V106A & wt & wt & wt & wt & wt & wt & wt & Yes & No \\
\hline
\end{tabular}

${ }^{a}$ AAC+AAT. AS-PCR, allele-specific real-time PCR; HIV-1, HIV type-1; ND, not done; NVP, nevirapine; NVP-SD, nevirapine single-dose; wt, wild type. 